DOI: $10.18027 / 2224-5057-2021-11-3 s 2-25$

Цитирование: Бредер В.В., Балахнин П.В., Виршке Э.Р., Косырев В.Ю., Ледин Е.В., Петкау В.В. Практические рекомендации по лекарственному лечению больных гепатоцеллюлярным раком. Злокачественные опухоли : Практические рекомендации RUSSCO \#3s2, 2021 (том 11). 25

\title{
ПРАКТИЧЕСКИЕ РЕКОМЕНДАЦИИ ПО ЛЕКАРСТВЕННОМУ ЛЕЧЕНИЮ БОЛЬНЫХ ГЕПАТОЦЕЛЛЮЛЯРНЫМ РАКОМ
}

Коллектив авторов: Бредер В.В., Балахнин П.В., Виршке Э.Р., Косырев В.Ю., Ледин Е.В., Петкау В.В.

Ключевые слова: гепатоцеллюлярный рак, фиброламеллярная карцинома, цирроз печени, BCLC, TAXЭ, РЧА, аблация, иммунотерапия, атезолизумаб, бевацизумаб, сорафениб, регорафениб, ленватиниб, кабозантиниб, рамуцирумаб, ниволумаб, ипилимумаб, гепатит С, гепатит

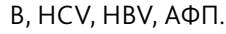

Гепатоцеллюлярный рак (ГЦР) - наиболее распространённая (около 85 \% случаев) злокачественная опухоль печени. Существенно реже встречаются холангиокарциномыопухоли из эпителия внутрипеченочных желчных протоков, смешанные гепатохолангиокарциномы, а также фиброламеллярная карцинома (ФЛК), которая, несмотря на иную молекулярно-генетическую структуру, классифицируется как вариант ГЦР. Описаны случаи синхронного и метахронного печеночно-клеточного и холангиоцеллюлярного рака.

ГЦР в большинстве случаев развивается на фоне хронических вирусных гепатитов В и/или С, цирроза печени любой этиологии, а также при некоторых наследственных заболеваниях (гемохроматоз, дефицит $\alpha 1$-антитрипсина, тирозинемия). Менее чем в $10 \%$ случаев ГЦР развивается в здоровой печени. Фиброламеллярная карцинома чаще всего выявляется у молодых пациентов, в т. ч. у подростков и не имеет известных факторов риска.

Заболевание, как правило, характеризуется длительным периодом внутрипеченочного роста, частым мультифокальным опухолевым поражением, что затрудняет раннюю диагностику на фоне диспластических и регенераторных узлов, наблюдаемых при циррозе печени. На рис. 1 схематично отражен наиболее частый вариант прогрессии ГЦР и возможное лечение.

Клиническая картина, как правило, имеет скрытый характер, не имеет специфических симптомов. Симптомокомплекс при ГЦР определяться фоновым заболеванием печени, циррозом, его осложнениями и осложнениями опухолевого процесса. Паранеопластические синдромы встречаются у небольшой части пациентов и проявляются гипогликемией, эритроцитозом, гиперкальциемией. 

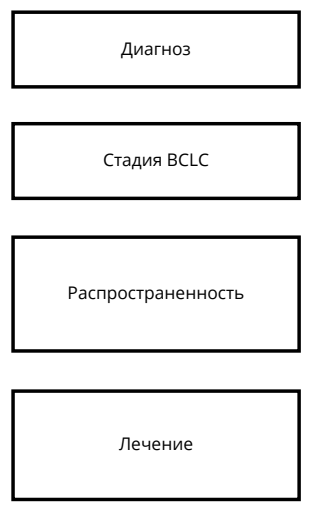

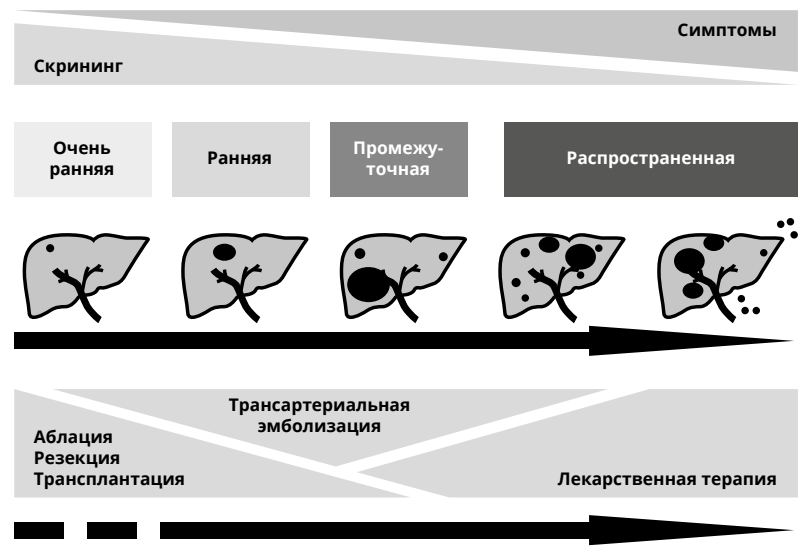

Рисунок 1. Клиническое течение ГЦР и варианты лечения.

\section{1. КЛАССИФИКАЦИЯ И ОПРЕДЕЛЕНИЕ СТАДИИ}

Для стадирования ГЦР применяются 8-я редакция классификации по системе TNM Международного противоракового союза AJCC/UICC, которая имеет практическое значение для операбельных случаев заболевания и классификация BCLC (Barcelona Clinic Liver Cancer или Барселонская система стадирования ГЦР).

\section{1. Классификация TNM/AJCC (8 пересмотр, 2017 г.)}

Тх - оценить первичную опухоль невозможно

ТО - признаков первичной опухоли нет

T1а - одиночная опухоль <2 см в наибольшем измерении, с сосудистой инвазией или без нее

$\mathrm{T} 1 \mathrm{~b}$ - одиночная опухоль > 2 см в наибольшем измерении, без сосудистой инвазии

Т2 - одиночная опухоль с сосудистой инвазией >2 см или множественные опухоли не более 5 см в наибольшем измерении

Т3 - множественные опухоли, в том числе одна опухоль > 5 см в наибольшем измерении

T4 - одиночная или множественные опухоли любого размера спрорастанием в крупнуюветвь воротной вены либо в печеночную вену, либо прорастание в соседние органы, включая диафрагму (кроме желчного пузыря), или прорастание в висцеральную брюшину

$\mathrm{N}$ - регионарные лимфатические узлы. Регионарными лимфатическими узлами являются лимфатические узлы ворот печени (расположенные в печеночно-двенадцатиперстной связке)

$\mathrm{Nx}$ - недостаточно данных для оценки состояния регионарных лимфатических узлов

$\mathrm{NO}$ - нет признаков метастатического поражения регионарных лимфатических узлов

$\mathrm{N} 1$ - имеется поражение регионарных лимфатических узлов метастазами 
Mх - недостаточно данных для определения отдаленных метастазов

Мо - отдаленных метастазов нет

M1 - имеются отдаленные метастазы.

PTNM - патогистологическая классификация.

Требования к определению категорий рT, pN, pM соответствуют требованиям к определению категорий T, N, M.

G - гистопатологическая дифференцировка:

$\mathrm{Gx}$ - степень дифференцировки не может быть установлена

$\mathrm{G} 1$ - высокая степень дифференцировки

$\mathrm{G} 2$ - средняя степень дифференцировки

$\mathrm{G} 3$ - низкая степень дифференцировки

G4 - недифференцированные опухоли.

Таблица 1. Группировка по стадиям ГЦР (АЈСС)

\begin{tabular}{|l|l|l|l|}
\hline Стадия & T & N & M \\
\hline IA & T1a & N0 & M0 \\
\hline IB & T1b & N0 & M0 \\
\hline II & T2 & N0 & M0 \\
\hline IIIA & T3 & N0 & M0 \\
\hline IIIB & T4 & N0 & M0 \\
\hline IVA & Любое значение T & N1 & M0 \\
\hline IVB & Любое значение T & Любое значение N & M1 \\
\hline
\end{tabular}

\section{2. Барселонская система стадирования}

BCLC (Barcelona Clinic Liver Cancer) - наиболее часто используемая и распространенная классификация ГЦР учитывает распространенность опухолевого процесса, функциональное состояния печени, объективное состояния больного и предполагаемую эффективность лечения (рис. 2). Выделяют пять стадий болезни: от стадии 0 - очень ранней и А - ранней до стадии D - терминальной. Стадия BCLC, равно как прогноз заболевания и тактика лечения, может меняться при прогрессировании заболевания, либо эффективном лечении. Следует отметить прогностическую значимость классификации для ГЦР безотносительно наличия/отсутствия цирроза печени. Важная особенность этой классификации в том, что в ней предложен алгоритм лечения в зависимости от стадии заболевания. В последней редакции этой классификации (2018 г.) вместо класса цирроза по Child-Pugh используется термин «сохранная функция печени», что может укладываться в нарушение функций печени, описываемых в пределах Child-Pugh A-B <8 баллов. С учетом высокой прогностической значимости классификации BCLC настоятельно рекомендуется ее использование как при локализованном, так и распространенном опухолевом процессе.

Очень ранняя (BCLC 0) - солитарная опухоль менее 2 см в диаметре. 
Ранняя стадия или BCLC A - это солитарная опухоль печени любого размера или не более 3 узлов максимальным размером до 3 см, не распространяющаяся на магистральные сосуды печени и соседние анатомические структуры при отсутствии опухолеспецифических жалоб, удовлетворительном объективном состоянии (ECOG =0) и сохранной функции печени.

Промежуточная стадия или BCLC B - это случаи изолированного бессимптомного множественного опухолевого поражения печени без макрососудистой инвазии, у больных в удовлетворительном состоянии (ECOG $=0)$ при сохранной функции печени.

Распространенная стадия или ВCLC C - симптомный ГЦР, ухудшающий объективное состояние (ECOG =1-2), с опухолью любого размера в сочетании или без инвазии магистральных печеночных сосудов и/или внепеченочным распространением и при сохранной функции печени.

Терминальная стадия или BCLC D - случаи заболевания с неудовлетворительным объективным состоянием (опухоль/цирроз), декомпенсированной функцией печени (Child-Pugh B/C $\geq 8$ баллов). Иногда, при выявлении малой опухоли (солитарная < 5 см или не более 3 опухолей размером наибольшей <3 см, т. н. «миланские критерии» возможна ортотопическая трансплантация печени).

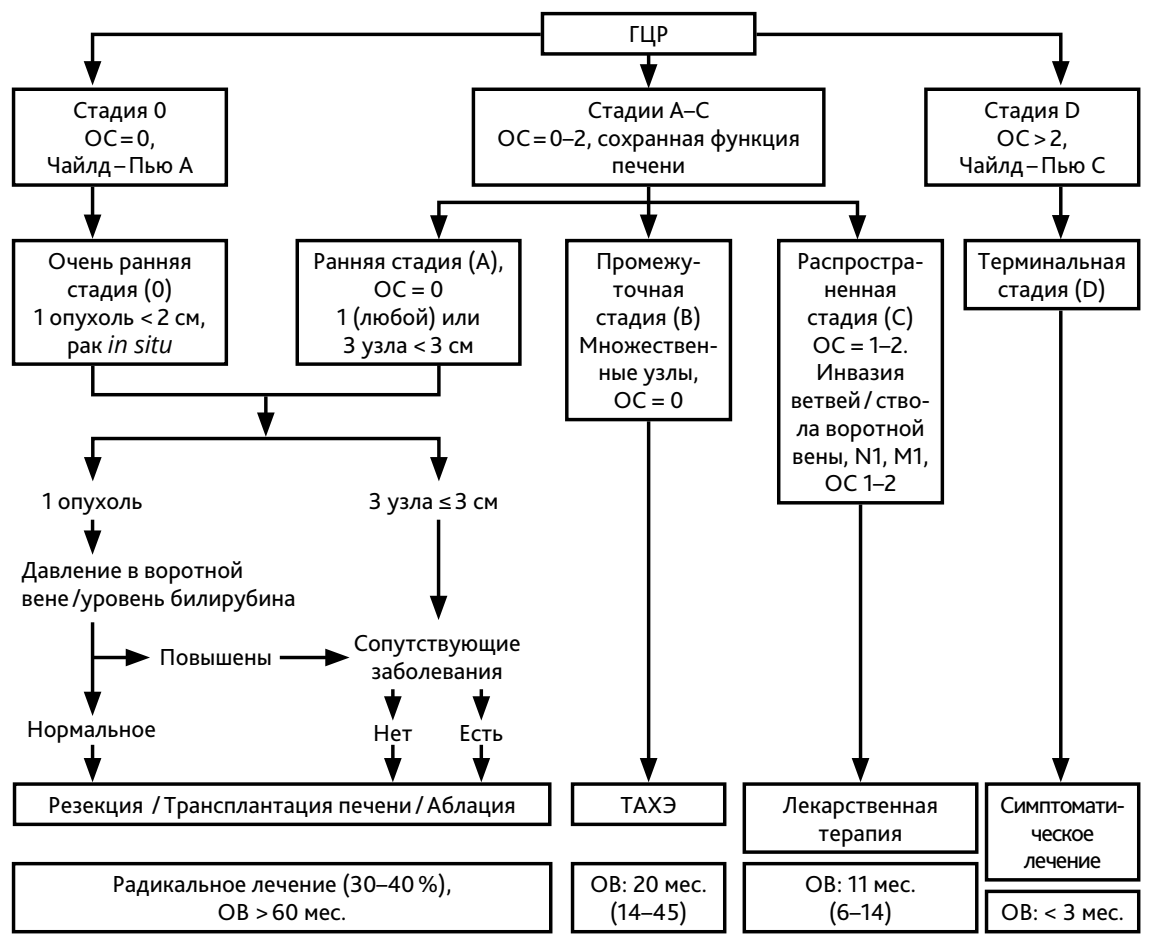

Pис. 2. BCLC или Барселонская система стадирования гепатоцеллюлярного рака. 


\section{2. ДИАГНОСТИКА}

Диагноз ГЦР устанавливается на основании патогномоничных клинико-рентгенологических данных, результатов патолого-анатомического исследования биопсийного или операционного материала, лабораторных исследований, направленных на оценку функции печени, и инструментального обследования распространенности опухолевого процесса и выраженности сопутствующих заболеваний.

Чаще всего ГЦР развивается на фоне хронического заболевания печени - цирроза печени, вирусного гепатита, стеатогепатита - которое, как и опухолевый процесс, может проявляться специфическими симптомами, нуждаться в специфической терапии, конкурентно влиять на качество жизни и ухудшать выживаемость. В рамках мультидисциплинарной оценки состояния пациента необходимо оценить функциональные резервы печени, причину и выраженность фоновой патологии печени, распространенность опухолевого процесса.

В группу риска развития ГЦР входят больные циррозом печени любой этиологии, носители вируса гепатита В, с семейным анамнезом ГЦР (для азиатов - мужчин старше 40 лет и женщин старше 50 лет); для них целесообразно регулярное (каждые 6 месяцев) УзИ печени и одновременное определение уровня АФП. Уровень онкомаркера считается позитивным при АФП > 100 нг/мл или увеличении его уровня на 7 нг/мл/мес. по результатам трехкратного ежемесячного измерения. При повышенном уровне АФП у пациента из группы риска развития ГЦР должны быть выполнены мультифазная контрастная КТ и контрастная МРТ печени и брюшной полости независимо от результатов УЗИ печени. Следует помнить, что только примерно в 20\% случаях уровень маркера значительно повышен (более чем 400 нг/мл), а почти в половине случаев концентрация маркера не превышает 20 нг/мл. Уровень АФП может повышаться при хронических заболеваниях печени, например, при острых и хронических вирусных гепатитах, при других онкологических заболеваиях (гепатоидный рак желудка, герминогенные опухоли и т. д.), беременности. При выявленной очаговой патологии в печени нормальный или слегка повышенный уровень АФП не влияют на дальнейший диагностический поиск.

При отсутствии опухолевых образований в печени пациента из группы риска рекомендуется повторное обследование каждые 3-6 месяцев (УЗИ и/или контрастное КТ/МРТ, АФП). Алгоритм обследования зависит от размеров образования в печени, наличия/отсутствия цирроза (рис. 3). Поскольку риск развития ГЦР коррелирует с длительностью существования и выраженностью цирроза печени при длительном наблюдении за пациентами из группы риска, циррозе печени с признаками портальной гипертензии следует отдавать предпочтение КТ и МРТ с контрастированием. 


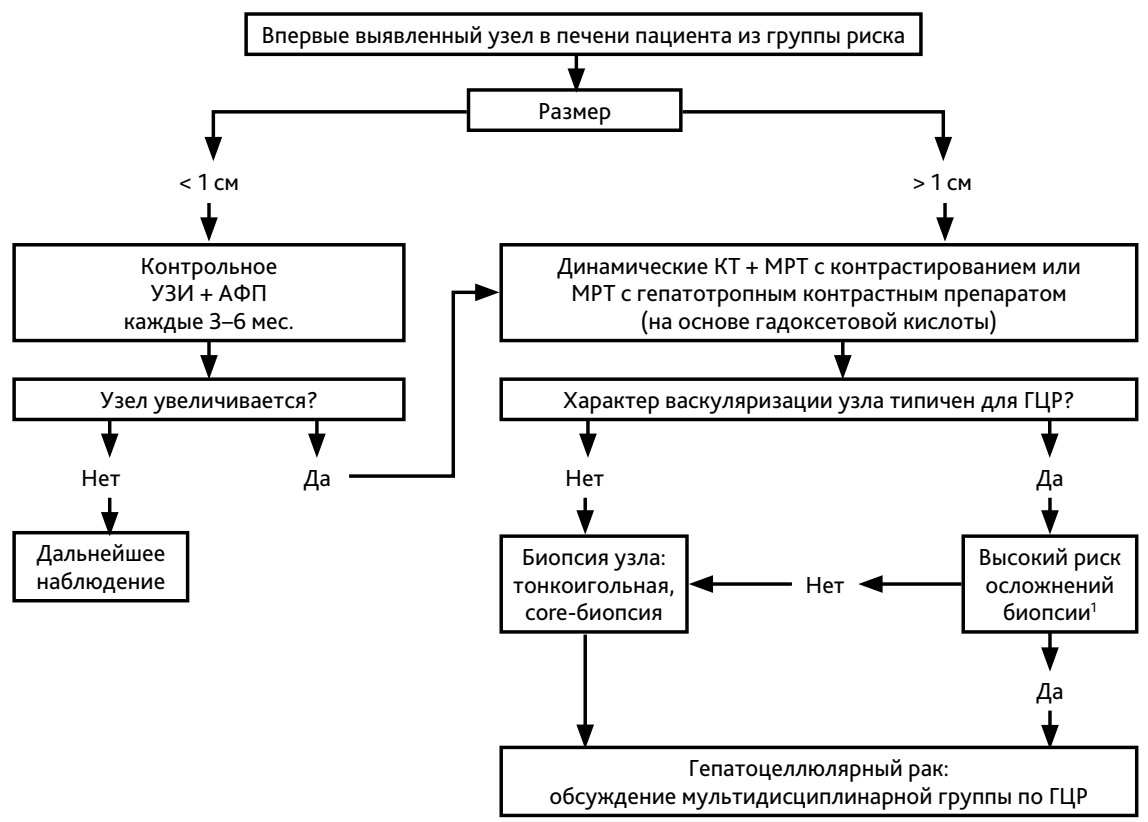

1 Диагноз ГЦР считается обоснованным без морфологической верификации, если в цирротически измененной печени динамическая контрастная КТ и МРТ выявляют типичную для ГЦР васкуляризацию опухоли).

Рисунок 3. Диагностический алгоритм для пациента из группы риска при подозрении на ГЦР.

Обследование направлено на уточнение распространенности опухолевого процесса, выраженности фоновой патологии печени и ее осложнений, объема и возможности проведения оптимального противоопухолевого лечения с учетом объективного состояния пациента и сопутствующей патологии. При наличии абсолютных противопоказаний к противоопухолевому лечению объем обследования определяется целями симптоматической терапии.

- Сбор жалоб и анамнеза, уточнение наличия факторов риска развития ГЦР

- Осмотр врачебный, определение объективного статуса пациента по шкале ЕСОG.

- Лабораторная диагностика: клинический (+тромбоциты) и биохимический (+альбумин, электролиты) анализы крови, АФП, коагулограмма (протромбиновое время, МНО), общий анализ мочи.

- Панель вирусных гепатитов.

- Определение поверхностного антигена к вирусу гепатита B (HBsAg)

- При HBsAg+: определение $\mathrm{HBeAg}, \mathrm{HBeAb}$ и количественное определение ДНК $\mathrm{HBV}$, консультация гепатолога для решения вопроса о противовирусной терапии. 
- Определение антител к ядерному антигену гепатита B (HBcAb) IgC

- Определение антител к гепатиту C (HCV), при положитетельном значении ? определение количества РНК в плазме, определение генотипа и консультация гастроэнтеролога.

- Оценка функционального статуса печени по классификации Child-Pugh (CP). Каждый признак в зависимости от выраженности оценивается в баллах от 1 до 3 (табл. 2). Сумма баллов 5-6, 7-9 и 10-15 классифицируются как СР класса А, В и С соответственно. Применение инструментов - калькуляторов объективной оценки функции печени, таких как ALBI или PALBI - возможно после валидации в крупных клинических исследованиях.

Таблица 2. Балльная система оценки функции печени Child-Pugh

\begin{tabular}{|l|l|l|l|}
\hline Характеристика & 16алл & 2 балла & 3 балла \\
\hline Энцефалопатия (степень) & Нет & $\begin{array}{l}\text { I-ІІ степень (или } \\
\text { компенсируется } \\
\text { медикаментозно) }\end{array}$ & $\begin{array}{l}\text { III-IV степень (или } \\
\text { рефрактерная) }\end{array}$ \\
\hline Асцит & Нет & «Незначительный» & «Умеренный» \\
\hline Альбумин плазмы (г/дл) & $>3,5$ & $2,8-3,5$ & $<2,8$ \\
\hline $\begin{array}{l}\text { Увеличение протромбинового времени, } \\
\text { сек. (выше N=12-16 сек.) или } \\
\text { МНО }\end{array}$ & $+1-4$ сек & $+4-6$ сек. & $>+6$ сек. \\
\hline Билирубин общий & $<1,7$ & $1,7-2,3$ & $>2,3$ \\
\hline
\end{tabular}

- Определение фоновой патологии печени (при наличии - оценка степени выраженности и прогноза для жизни);

- при циррозе и вирусных гепатитах: консультация гастроэнтеролога для уточняющей диагностики возможных осложнений гепатитов и цирроза, прогноза течения и подбора противовирусной/сопроводительной терапии.

- УзИ органов брюшной полости и забрюшинного пространства

- УзИ печени с контрастированием - высокоинформативный метод уточняющей диагностики раннего ГЦР в цирротической печени в условиях высокоспециализированного центра; обязательна оценка состоятельности кровотока в бассейне воротной вены.

- КТ, 4-фазная (нативная, артериальная, венозная и отсроченная венозная фазы) и/или динамическая МРТ органов брюшной полости с в/в контрастированием.

- Диагноз ГЦР считается обоснованным без морфологической верификации, если в цирротически измененной печени динамическая контрастная КТ и МРТ выявляют типичнуюдля ГЦР васкуляризацию в опухоли. МРТ выполняется с гепатоспецифическим контрастным препаратом гадоксетовой кислоты для выявления раннего ГЦР (до 2,0 см) у пациентов с сопутствуюим циррозом печени любой этиологии. Диагностика раннего ГЦР и своевременно начатое лечение имеют решающее 
значение для улучшения прогноза и качества жизни пациентов, повышая долгосрочную выживаемость. Особенности накопления и вымывания контрастного препарата из опухолевых тканей в артериальную, венозную и отсроченные фазы при использовании экстрацеллюлярных магнитно-резонансных контрастных препаратов в ряде случаев не позволяют выявлять и корректно дифференцировать новообразования, в том числе малых размеров. Гиперинтенсивность печеночной паренхимы в гепатоспецифическую фазу позволяет отчетливо визуализировать гипоинтенсивные очаги малых размеров и, с учетом особенностей их контрастирования в предыдущие фазы исследования, дифференцировать ранний ГЦР от прочих новообразований печени, в т. ч. и от регенераторных и диспластических узлов, что в ряде случаев может повлиять на изменение стадии опухолевого процесса.

- МРТ печени с гепатоспецифическим контрастным препаратом гадоксетовой кислоты для корректного стадирования опухолевого процесса перед началом лечения имеет преимущества по сравнению с другими методами. В ряде систематических обзоров рандомизированных клинических исследований с применением мета-анализа доказана высокая информативность МРТ с применением гепатоспецифического контрастного препарата гадоксетовой кислоты по сравнению с КТ и МРТ с использованием экстрацеллюлярных контрастных препаратов в выявлении и дифференциальной диагностике ГЦР, что позитивно отражается на общей выживаемости.

- Морфологическое подтверждение диагноза: пункционная биопсия опухоли печени (тонкоигольная или с получением столбика ткани - core-биопсия, предпочтительна для дифференциальной диагностики):

- при малом размере опухоли (<2 см) и типичном для ГЦР кровотоке

- при нетипичной васкуляризации узла размером > 2 см

- если квалифицированные рентгенологи расходятся в описании и трактовке контрастных динамических исследований (РКТ/МРТ) в сочетании с нормальным или незначительно повышенным уровнем АФП

- в случае выявления любого опухолевого образования в нецирротической печени; - для уточняющей диагностики раннего и высокодифференцированного ГЦР целесообразна консультация гистопрепаратов в специализированных центрах; - при технической невозможности морфологической верификации диагноза следует предпринять дополнительный диагностический поиск с учетом рекомендаций врачебного консилиума

- Диагноз печеночно-клеточного рака (ГЦР) считается обоснованным без морфологической верификации, если в цирротически измененной печени по данным одного из методов (мультифазной КТ или МРТ) выявляют типичные для ГЦР признаки, а именно диффузное (не кольцевидное) контрастное усиление опухоли размером >1 см в поздней артериальной фазе и «вымывание» контрастного вещества в венозной фазе; в опухолевых узлах размером >2 см выявление псевдокапсулы в отсроченной (равновесной) фазе; рост опухоли <6 мес. на 50\% или увеличение размеров опухоли на 25 мм за 6 мес.

- Рентгенография органов грудной клетки

- КТ органов грудной клетки. 
- ЭГДС: наличие/степень варикозного расширения вен пищевода/желудка, эрозивноязвенных изменений.

- ЭКГ.

- Остеосцинтиграфия при подозрении на метастатическое поражение скелета.

- КТ-ангиография печени в сложных случаях при планировании хирургического лечения; является также неотъемлемой составной (первый этап) частью химиоэмболизации опухолей печени.

- ПЭТ-КТ (с холином, ФДГ). ПЭТ-КТ с глюкозой (ФДГ) обладает низкой чувствительностью (на ПЭТ-позитивный ГЦР приходится только $40 \%$ случаев) и специфичностью. Повышенное значение SUV говорит об агрессивности процесса и предполагает низкую эффективность локальных видов лечения. ПЭТ-КТ с холином может быть эффективна для выявления внепеченочных метастазов.

- МРТ или КТ головного мозга с в/в контрастированием при симптомах очагового поражения

- В результате проведенного обследования должна быть сформулирована стадия опухолевого процесса согласно принятым классификациям TNM (8 пересмотра)/AJCC и BCLC (Барселонская классификация), определен функциональный класс печени по классификации Child-Pugh, определены показания и противопоказания к противоопухолевому лечению.

\section{3. ЛЕЧЕНИЕ}

План лечения учитывает наличие и степень цирроза, распространенность опухолевого процесса, функциональные резервы печени, объективное состояние больного и прогноз основного и фонового заболевания печени, а также сопутствущую патологию и пожелания пациента. Наилучшие результаты получены при обсуждении результатов диагностики и определении плана лечения мультидисциплинарной группой по ГЦР на базе специализированных центров. В лечении ГЦР применяются следующие опции:

- хирургическое лечение (3.1)

- резекции печени (3.1.1)

- ортотопическая трансплантация печени (3.1.2)

- локальная деструкция опухоли (3.2)

- энергетическая деструкция (радиочастотная, микроволновая, криоаблация) (3.2.1)

- инъекция этанола (3.2.2)

- стереотаксическая Лт (3.2.3)

- регионарная терапия (3.3)

- трансартериальная химиоэмболизация (3.3.1):

- масляная ТАХЭ (3.3.1.1)

- ТАХЭ элиминирующими микросферами DEB (3.3.1.2)

- комбинированная (этапная) ТАХЭ (3.3.1.3)

- трансартериальная радиоэмболизация (3.3.2)

- системная лекарственная терапия (3.4). 


\section{1. Оперативное лечение}

\subsection{1. Резекция печени с достижением RO}

Метод выбора лечения раннего ГЦР без сопутствующего цирроза печени. Резекция возможна и у больных с циррозом при сохранной функции печени, нормальном уровне билирубина и без признаков портальной гипертензии. Размер опухоли и множественное внутриорганное поражение не являются абсолютным противопоказанием к резекции, однако результаты хирургического лечения в этих случаях предсказуемо хуже.

\subsection{2. Ортотопическая трансплантация печени}

Метод выбора лечения раннего ГЦР (BCLC 0/A) в случаях, не подходящих для резекции или при декомпенсированном (Child-Pugh B/C) циррозе печени с учетом принятых критериев трансплантабельности. При определении показаний к трансплантации, как правило, используются так называемые «Миланские критерии»: размер единственной опухоли не более 5 см или наличие в печени до 3 очагов с диаметром наибольшего узла не более 3 см и отсутствием инвазии в сосуды.

Пациенты, ожидающие трансплантацию печени, могут получать как неоадъювантное, так и иное противоопухолевое лечение (bridge-терапия, или терапия «ожидания»), в том числе аблацию, химиоэмболизацию, резекцию печени, и/или системное лечение, что увеличивает вероятность выполнения трансплантации печени, способствует селекции и отбору менее агрессивных случаев ГЦР. При успешном консервативном лечении случаев местно-распространенного ГЦР, исходно не подходивших под критерии трансплантации и рентгенологически подтвержденном «понижении» стадии (down-staging) до принятых критериев трансплантабельности, целесообразно рассмотреть вопросо трансплантации печени как методе радикального лечения ГЦР и цирроза.

\subsection{3. ААъювантная противоопухолевая терапия}

После радикального (RO) хирургического лечения адъювантная терапия мультикиназными ингибиторами не рекомендуется в связи с отсутствием преимуществ перед другими вмешательствами в улучшении выживаемости пациентов.

- После радикального лечения ранних стадий ГЦР при сопутствующем хроническом вирусном гепатите B (HBs+, вирусная нагрузка >10000 копий в мл) рекомендуется противовирусная терапия аналогами нуклеозидов; она увеличивает безрецидивную и общую выживаемость.

- После радикального лечения пациентам с ГЦР и сопутствующем хроническим гепатите C (РНК-ВГС+) рекомендуется противовирусная терапия ингибиторами протеазы и полимеразы вируса (глекапревир/пибрентасвир или софосбувир/велпатасвир при компенсированном поражении печени) или только ингибиторами полимеразы вируса (софосбувир/велпатасвир при декомпенсированном поражении печени [класс В или C по Child-Pugh]), после радикального лечения ранних стадий ГЦР она увеличивает безрецидивную и общую выживаемость. При нерадикальном хирургическом лечении 
после периода восстановления пациента рекомендуется системная терапия 1 линии (см. Системное лекарственное лечение).

\section{2. Методы локальной деструкции опухоли}

В качестве методов локальной деструкции (энергетической аблации) рекомендуется использовать радиочастотную аблацию, микроволновую аблацию и криоаблацию. В качестве метода локальной деструкции (химической абляции) рекомендуется использовать чрескожное введение этилового спирта.

\subsection{1. Энергетическая деструкция малых (до 3 см в диаметре) узлов ГЦР}

Применяется у пациентов, не подлежащих хирургическому лечению. Используется система гипертемического (радиочастотная, микроволновая, др.) либо гипотермического (криоаблация) воздействия. Вид доступа (интраоперационный, лапароскопический или чрескожный) в каждом случае определяется исходя из конкретной клинической ситуации, оснащенности лечебного учреждения и навыков оператора.

Решение о целесообразности деструкции должно приниматься мультидисциплинарной комиссией, в составе профильного хирурга, химиотерапевта, радиолога и интервенционного радиолога (рентгенохирурга). Если принимается положительное решение о необходимости такого лечения, то дальнейшее планирование тактики терапии осуществляется непосредственно интервенционным радиологом. Основным критерием при определении показаний является техническая возможность выполнения вмешательства с минимальным риском развития осложнений. Деструкции подлежат не более 5 узлов диаметром $\leq 3$ см. Возможна деструкция узлов большего диаметра, но частота локальных рецидивов существенно увеличивается при опухолях >3 см в наибольшем измерении. В ряде случаев целесообразно выполнять аблацию после проведения предварительной трансартериальной (химио -) эмболизации опухоли печени или осуществлять энергетическую аблацию в комбинации с чрескожным введением этилового спирта (химическая аблация).

Локальная деструкция противопоказана при множественном поражении печени, декомпенсированном циррозе (класса C по Child-Pugh), внепеченочных проявлениях заболевания, при наличии портального шунта, несмещаемом прилежании опухолевого узла к рядом расположенным полым органам, а также к вне (внутри -) печеночно расположенным трубчатым структурам.

Рекомендуется применять методы локальной деструкции (энергетической аблации) опухоли как стандарт терапии у пациентов с ГЦР стадии BCLC 0 (солитарная опухоль до 2 см в диаметре) и стадии BCLC A (три опухоли до 3 см в диаметре) при невозможности выполнения хирургического лечения (резекции печени или трансплантации печени).

Возможно применение методов локальной деструкции (энергетической и химической аблации) в сочетании с другими видами лечения, в том числе с внутриартериальной терапией, системной терапией и хирургическим лечением и в случаях локального прогрессирования и локального рецидива заболевания (если это технически возможно), 
после ранее выполненной резекции печени или абляции опухоли при невозможности выполнения хирургического лечения.

Отдаленные результаты применения энергетической аблации опухолей менее 3 см, по данным рандомизированных исследований и мета-анализов, не хуже, чем результаты резекции печени.

Оценка локального ответа на термическое воздействие выполняется по критериям MRECIST по КT или MPT с контрастным усилением либо непосредственно после завершения вмешательства либо через 4-6 недель после вмешательства. При успешном выполнении процедуры зона аблации должна выходить за визуализируемуюграницу опухоли не менее чем на 10 мм (в таком случае вероятность развития локального рецидива в зоне деструкции минимальна). В случае подозрения на наличие остаточной опухолевой ткани в зоне воздействия возможно повторное вмешательство и обсуждение дополнительных методов лечения - ТАХЭ и/или системной терапии.

\subsection{2. Чрескожная инъекция этанола в опухолевый узел}

Метод лечения очень раннего (BCLC 0, солитарная опухоль до 2 см в диаметре) ГЦР на фоне цирроза печени при невозможности проведения энергетической деструкции, например, вследствие специфической анатомической локализации узла (прилежание к крупным желчным протокам, желчному пузырю или кишке), как метод терапии при невозможности выполнения хирургического лечения (резекции печени или трансплантации печени, или как дополнение к методам энергетической аблации при лечении опухолей диаметром более 3 см и опухолей, прилежащих к крупным сосудам (у неоперабельных больных).

\subsection{3. Стереотаксическая лучевая терапия или стереотаксическая радиохирургия ГЦР}

Является приемлемым методом лечения раннего ГЦР (классификация BCLC). Кандидатами для стереотаксической ЛТ являются пациенты, подходящие для локального лечения при отсутствии внепеченочного поражения, ограниченном объеме поражения (не более 3 очагов), удовлетворительной функции печени и при невозможности выполнить другой вариант воздействия. Для опухолей более 2 см стереотаксическая ЛТ может обеспечивать локальный контроль, сравнимый с термической деструкцией опухоли.

\section{3. Регионарная терапия}

Метод применяется в качестве паллиативного лечения пациентов ГЦР при нерезектабельном/неоперабельном процессе без признаков инвазии/тромбоза магистральных печеночных сосудов и внепеченочных проявлений заболевания в комбинации с другими методами локального и системного воздействия, в период ожидания трансплантации печени, при изолированном внутрипеченочном прогрессировании после оперативного лечения. Согласно классификации BCLC, это случаи промежуточной стадии (BCLC B) и ранней стадии (BCLC A) при противопоказаниях к хирургическому лечению. Проведе- 
ние ТАХЭ/ТАРЭ опухоли в печени при наличии внепеченочных проявлений опухолевого процесса не улучшает отдаленных результатов лечения.

\subsection{1. Трансартериальная химиоэмболизация опухоли(ей) печени (TAXЭ)}

Важными критериями селекции пациентов для данного вида лечения являются общее состояние пациента не хуже ECOG0-1, компенсированные сопутствующие заболевания и переносимость эмболизации (одномоментной или поэтапной) всех узлов. Прогностически более благоприятной группой больных, которым показана ТАХЭ, являются пациенты с узловой формой поражения без цирроза печени, либо с циррозом Child-Pugh A, без инвазии опухоли в сосуды.

ТАХЭ выполняется при условии технической возможности (определяется интервенционным радиологом) и онкологической целесообразности (определяется на мультидисциплинарном обсуждении) вмешательства. С учетом современных возможностей системной терапии следует отдавать предпочтение селективному воздействию с целью минимизации нежелательного повреждения при ТАХЭ паренхимы печени и сохранения функциональных резервов органа. Выделяют следующие противопоказания к проведению ТАХЭ печени при ГЦР:

- абсолютные:

- декомпенсированный цирроз (Child-Pugh C);

- поражение более $75 \%$ объема печени;

- тромбоз ствола воротной вены;

- наличие артерио-венозной печеночной фистулы, не подлежащей окклюзии;

- почечная недостаточность (креатинин 2 мг/дл или клиренс креатинина <30 мл/мин.)

- относительные:

- размер опухоли > 10 см;

- некомпенсированные сопутствующие заболевания, включая нарушения функций органов сердечно-сосудистой, легочной системы, острые инфекционные заболевания, состоявшееся желудочно-кишечное кровотечение в срок до 3 мес.;

- варикозное расширение вен пищевода/желудка 3 степени с высоким риском кровотечения;

- желчная гипертензия.

Выбор метода ТАХЭ при ГЦР (масляная - раствор цитостатика +липиодол; комбинированная - раствор цитостатика +липиодол +микросферы из поливинилацетата; эмболизация микросферами, элиминирующими цитостатик (DEB - drug-eluting beads)) зависит от клинической ситуации, технических условий и плана лечения. Наиболее часто используемые цитостатические препараты - доксорубицин или эпирубицин, цисплатин. Оценка эффективности ТАХЭ проводится через 1 месяц по КТ/МРТ с в/в контрастным усилением с использованием критериев MRECIST. 


\subsubsection{1. Масляная ТАХЭ}

Применяется при многоузловом моно-либо билобарном поражении объемом не более $70 \%$ паренхимы печени:

- при многоузловом билобарном поражении ТАХЭ выполняется в два этапа (раздельная подолевая ТАХЭ с интервалом 4 недели, далее - интервал 6-8 недель до следующей ТАХЭ);

- интервалы между курсами одномоментной ТАХЭ - 6-8 недель;

- кратность введения - не менее 3 курсов (при сохранной функции печени и отсутствии внепеченочных проявлений заболевания).

\subsubsection{TAXЭ элиминирующими микросферами DEB (drug-eluting beads)}

Выполняется при моно-либо мультинодулярном, монолобарном поражении объемом не более $50 \%$ паренхимы печени. При сохранении васкуляризации в опухоли после сеанса TAХЭ (частичный некроз) выполняется повторная ТАХЭ (по той же схеме) с оценкой эффективности через 4 недели.

\subsubsection{3. Комбинированная (этапная) ТАХЭ}

- вариант A:

1 этап - DEB (суперселективная TAXЭ зоны доминантного поражения) +2 этап масляная селективная ХЭ зоны, не подвергшейся воздействию на 1-м этапе;

- вариант Б:

При моно-либо мультинодулярном, монолобарном поражении объемом не более $50 \%$ паренхимы печени.

1 этап - DEB (суперселективная TAХЭ зоны доминантного поражения) +2 этап - DEB селективная ТАХЭ зоны, не подвергшейся воздействию на первом этапе. Последующая тактика определяется эффективностью (mRECIST) первой TAXЭ по данным КТ и/или МРТ с контрастированием через 4 недели:

- при отсутствии контрастирования (тотальный некроз) в зоне воздействия ТАХЭ повторная ТАХЭ не показана; далее КТ и/или МРТ с контрастированием через каждые 6-8 недель.

- при сохранении васкуляризации в опухоли (частичный некроз) в зоне воздействия повторно выполняется ТАХЭ с контролем по данным КТ и/или МРТ с контрастированием через 4 недели.

Отсутствие эффекта ТАХЭ после 2 последовательно выполненных сеансов (нет зоны некроза в эмболизированных узлах, появление новых узлов в зоне воздействия) являются показанием к смене лечения и, как правило, к началу системной противоопухолевой терапии.

Повторная ТАХЭ при изолированном внутрипеченочном поражении оправдана при длительности эффекта ранее выполненной ТАХЭ более 6 месяцев при условии сохранной функции печени. 


\subsection{2. Трансартериальная радиоэмболизация (ТАРЭ)}

Заключается в инъекции в печеночную артерию радиоизотопа, заключенного в стеклянные или резиновые 5-миллиметровые микросферы. Данная опция обеспечивает локальное облучение опухоли с СОД 80-150 Гр, что приводит к некрозу ткани при приемлемой токсичности за счет селективного подведения короткоживущего радиоизотопа. ТАРЭ может использоваться при BCLC A в качестве bridge-терапии, BCLC B и C с тромбозом магистральных вен. Применение Иттрий -90-микросфер приводит к увеличению медианы времени без прогрессирования по сравнению с ТАХЭ с 6,8 до 26 мес., что, однако, не отражается на общей выживаемости: медиана продолжительности жизни при BCLC B составляет 16,9-17,2 месяцев, а при BCLC C 10-12 месяцев. В настоящее время нет четких критериев по выбору методики региональной терапии (ТАРЭ или ТАХЭ) и понимания, какой пациент получит большую пользу от того или иного метода. В целом, уровень объективного ответа на ТАРЭ составляет 30-50\%, время до прогрессирования - 6-26 мес. в зависимости от стадии и тромбоза портальной вены. Возможно повторное применение.

Основные противопоказания к ТАРЭ: поражение более $70 \%$ печени, наличие артериовенозного шунта, декомпенсированный цирроз (Child-Pugh C), лТ с вовлечением печени в анамнезе.

\section{4. Системное лекарственное лечение}

ГЦР - опухоль, устойчивая к терапии, поэтому рекомендуется в первую очередь рассмотреть возможность лечения в рамках клинических исследований (clinicaltrials.gov).

В лекарственном лечении ГЦР используются ингибиторы внутриклеточных киназ, механизм действия которых реализуется через антипролиферативное и антиангиогенное воздействие, иммунотерапия препаратами МКА, путем восстановления иммунного надзора над опухолью через блокаду рецепторов иммунотолерантности PD1/PD-L1 и CTLA4 и MKA, направленные на опухолевый ангиогенез. Использование цитостатиков ограничено их низкой эффективностью и сопряжено с высокой токсичностью на фоне сниженных функциональных резервов цирротически измененной печени. Для ГЦР в настоящее время не выявлены факторы прогноза эффективности иммунотерапии, ингибиторов протеинкиназ и цитотоксической ХТ.

Показаниями к системной лекарственной терапии при ГЦР являются:

- внепеченочное метастазирование и/или прогрессирование процесса в печени после применения локальных методов лечения;

- нецелесообразность (рассмотрение мультидисциплинарным консилиумомпотенциального клинического риска-пользы) или противопоказания для применения локальных методов лечения (резекции, трансплантации печени, ТАХЭ);

- внутрипеченочный локализованный рак - как терапия ожидания трансплантации печени (bridge-терапия).

В отдельных случаях системная лекарственная терапия ГЦР может сочетаться с ТАХЭ. Пациенты, являющиеся кандидатами для системного лечения, должны быть подробно информированы о целях и задачах, возможных побочных эффектах и особенностях тера- 
пии, необходимости контрольных обследований, возможной коррекции сопроводительного лечения; обязательно согласие пациента на планируемое лечение. Кандидат на лечение должен быть в относительно удовлетворительном общем состоянии (по шкале ECOG 0-2 балла), иметь сохранную функцию печени (Child-Pugh A/B - 5-7 баллов) и не страдать другой некомпенсированной сопутствущей патологией. Некомпенсированная кардиальная патология, недавние ишемические или тромбоэмболические нарушения, применение препаратов с антиангиогенным действием сопряжено с высоким риском осложнений. Опухолевый тромбоз печеночных вен и/или тромбоз ветвей/ствола воротной вены не являются противопоказанием к системной терапии ГЦР.

Помимо этого, основными условиями для проведения лекарственной терапии являются лабораторные показатели крови: общий билирубин < 51 мкмоль/л; АСТ /АЛТ < 5 значений ВГН; МНО <1,7; тромбоциты >50,0×10\%/л (только для терапии мультикиназными ингибиторами); креатинин <1,5 значений ВГН; альбумин >28г/л; отсутствуют электролитные нарушения.

Особое внимание следует уделить пациентам с сопутствущей артериальной гипертонией. Терапия мультикиназными ингибиторами и блокаторами ангиогенеза может быть начата при условии эффективного (повышение АД не выше 1 степени) гипотензивного лечения. Подробный алгоритм коррекции токсических проявлений изложен в инструкции к препаратам.

\subsection{1. Первая линия терапии ГЦР}

В качестве предпочтительной первой линии системной терапии ГЦР рекомендуется комбинация PD-L1-ингибитора атезолизумаба в дозе 1200 мг и моноклонального антиVEGF антитела бевацизумаба в дозе 15 мг/кг внутривенно 1 раз в 21 день для улучшения контроля роста опухоли и выживаемости пациентов (табл. 3). Комбинация атезолизумаба и бевацизумаба по данным рандомизированного клинического исследования 3 фазы (ImBrave150) продемонстрировала статистически значимое преимущество перед сорафенибом по общей выживаемости (медиана - 19,2 мес. vs. 13,4 мес.) и выживаемости без прогрессирования (медиана - 6,9 мес. vs. 4,3 мес.). Относительными противопоказанием к терапии являются аутоиммунные заболевания, активная ко-инфекция вирусами гепатита В и С, состоявшееся кровотечение или высокий риск кровотечения из варикозно-расширенных вен пищевода и желудка.

В качестве альтернативы, при наличии противопоказаний к назначению иммунотерапии в первой линии лечения ГЦР рекомендуются мультикиназные ингибиторы протенкиназ сорафениб или ленватиниб (табл. 3), статистически значимо увеличивающих выживаемость больных распространенным ГЦР.

Лечение проводится непрерывно, до объективных (КТ/МРТ) признаков прогрессирования заболевания, непереносимой токсичности или декомпенсации сопутствующих заболеваний.

Начальная суточная доза сорафениба - 800 мг, в случае цирроза Child-Pugh B (7 баллов) и для ослабленных пациентов или при выраженных сопутствующих заболеваниях -400 мг/день, при удовлетворительной переносимости возможна эскалация дозы 
до 800 мг/день. При развитии выраженной ( $\geq 2$ степени) специфической токсичности доза может быть снижена до 600/400/200 мг.

Ленватиниб рекомендован пациентам с функцией печени не хуже Child-Pugh A (6 баллов). Дозовый режим применения ленватиниба зависит от исходного веса пациента: для больных с массой тела $\geq 60$ кг лечение начинается с дозы 12 мг в день однократно, для пациентов с массой < 60 кг - с разовой суточной дозы 8 мг. При развитии выраженной ( $\leq 2$ степени) специфической токсичности доза ленватиниба снижается на 4 мг.

У пациентов с начальными признаками декомпенсации цирроза печени или при противопоказаниях к использованию ингибиторов протеинкиназ в качестве альтернативы (Child-Pugh A/B, 5-7 баллов) рекомендуется иммунотерапия ниволумабом для улучшения контроля роста опухоли и выживаемости пациентов в одном из рекомендованных режимов лечения (табл. 3).

\subsection{2. Вторая линия терапии ГЦР}

Представлена несколькими опциями (табл. 3): мультикиназными ингибиторами - регорафенибом и кабозантинибом, обладающими антипролиферативным и антиангиогенным эффектами, ингибитором ангиогенеза рамуцирумабом и блокаторами контрольных точек иммунного надзора (MKA к PD1/L1), включая комбинированную иммунотерапию; выбор последующего лечения определяется вариантом первой линии терапии, ее переносимостью, объективным состоянием пациента (не более ЕСOG 0-1), выраженностью нарушений функции печени Поскольку все известные рандомизированные плацебо-контролируемые исследования 2-3 линии лечения распространенного ГЦР выполнены в популяции больных, получавших в качестве 1 линии сорафениб, на сегодня нет данных об эффективности других опций после иммунотерапии или ленватиниба. Поэтому выбор 2 линии терапии определяется ранее использованным вариантом лечения:

а) после иммунотерапии рассматриваются ингибиторы протеинкиназ сорафениб или ленватиниб, регорафениб или кабозантиниб или рамуцирумаб (при уровне АФП > 400 нг/мл), эффективность комбинированной иммунотерапии неизвестна, эффективность рамуцирумаба после использования бевацизумаба неизвестна;

б) после ингибиторов протеинкиназ могут использоваться регорафениб, кабозантиниб, рамуцирумаб или иммунотерапия.

При прогрессировании на ленватинибе также возможен переход на сорафениб. При плохой переносимости сорафениба к моменту окончания 1 линии терапии (<400 мг/день) регорафениб или рамуцирумаб не применяются.

- Регорафениб - мультикиназный ингибитор рекомендован больным с прогрессированием на сорафенибе (при условии переносимости сорафениба в дозе $\geq 400$ мг/день) в удовлетворительном общем состоянии (ECOG 0/1) и при компенсированной функции печени (Child-Pugh A). Назначение регорафениба возможно через 1-2 недели после окончания сорафениба в стандартном (160 мг/день однократно 1-21 день, курс 4 недели) режиме или в начальной дозе 80-120 мг/день (1-21-й дни 4 недельного цикла) для пациентов, имеющих факторы риска развития токсичности (сорафениб 400 мг/день в анамнезе). При начале лечения в сниженной дозе в отсутствие токсичности $\geq 2$ сте- 
пени через 10-12 дней от начала курса оправдана эскалация суточной дозы препарата до стандартно рекомендуемой (160 мг/день). Поскольку регорафениб имеет схожий с сорафенибом профиль токсичности рекомендации по ведению больных совпадают.

- Кабозантиниб - мультикиназный ингибитор - назначается в дозе 60 мг внутрь 1 раз в день ежедневно длительно пациентам в удовлетворительном состоянии (ECOG 0/1) и при компенсированной функции печени (Child-Pugh A). Непереносимость сорафениба не является противопоказанием к назначению кабозантиниба. Лечение продолжают до тех пор, пока сохраняется клиническая польза от терапии или до развития неприемлемой токсичности. При развитии нежелательных реакций может потребоваться снижение дозы до 40 или 20 мг в день вплоть до прекращения терапии.

- Рамуцирума6 - MKA к VEGFR 2 типа назначается пациентам с уровнем АФП $\geq 400$ нг/мл при прогрессировании ГЦР или непереносимости терапии сорафенибом. Препарат назначается пациентам, в удовлетворительном состоянии (ECOG 0/1), при компенсированной функции печени (Child-Pugh A), удовлетворительно переносившим терапию сорафенибом ( $\geq 400$ мг/день) в дозе 8 мг/кг/внутривенно каждые 2 недели до прогрессирования или непереносимой токсичности больным.

Поскольку токсичность, ассоциированная с применением мультикиназных ингибиторов (диарея, артериальная гипертония, ладонно-подошвенный синдром) является биологическим предиктором лучшей выживаемости при условии продолжения терапии, целесообразно предпринять все меры по коррекции токсичности, в первую очередь - своевременное снижение суточной дозы препарата. При развитии токсичности 3-4 степени необходимы перерыв в приеме препарата на 1 неделю и активная симптоматическая терапия. При уменьшении токсичности <2 степени терапия возобновляется в редуцированной дозе и на фоне подобранной терапии.

В качестве 2-3 линий лечения прогрессирующего ГЦР или при непереносимости терапии киназными ингибиторами сорафенибом/ленватинибом/регорафенибом/кабозантинибом рекомендуется иммунотерапия препаратами МКА к рецепторам иммунотолерантности PD1/PD-L1 и CTLA4 или комбинации атезолизумаб/бевацизума6.

В настоящее время не известны предикторы эффективности иммунотерапии ГЦР. Иммунотерапия ниволумабом назначается в одном из дозовых режимов (табл. 3); пембролизумабом - по 200 мг в/в 1 раз в 3 недели до клинически значимого прогрессирования опухоли (табл. 3).

Комбинация ниволумаба (1 мг/кг) и ипилимумаба (3 мг/кг) (табл. 3) в нерандомизированном исследовании CheckMate-040 при лечении сорафениб-рефрактерного ГцР продемонстрировала высокую эффективность (8\% полных и 24\% частичных эффекта) при медиане продолжительности жизни 22,8 мес. и удовлетворительной переносимости.

Моноиммунотерапия может быть методом выбора лечения распространенного ГЦР в первой и последующих линиях лечения при нарушенной функции печени (класс цирроза Child-Pugh B (5-8 баллов) и/или при противопоказаниях к мультикиназным ингибиторам и рамуцирумабу; может использоваться иммунотерапия ниволумабом в стандартных дозах; пока нет данных о возможности применения пембролизумаба. Нет данных о негативном влиянии иммунотерапии на течение цирроза печени. Вероятность реактивации вирусных 
гепатитов низкая, хотя в случаях HBsAg+ необходима одновременная противовирусная терапия аналогами нуклеозидов на всем протяжении противоопухолевого лечения.

Цитотоксическая химиотерапия ГЦР эффективна в 20\% случаев, существенно не увеличивает продолжительности жизни, но в ряде случаев может рассматриваться в качестве лечебной опции у больных ГЦР без цирроза, например, при фиброламеллярной карциноме, в качестве второй-третьей линии терапии. Применяются платиносодержащие режимы в сочетании с гемцитабином (табл. 3).

Таблица 3. Режимы лекарственного лечения гепатоцеллюлярного рака

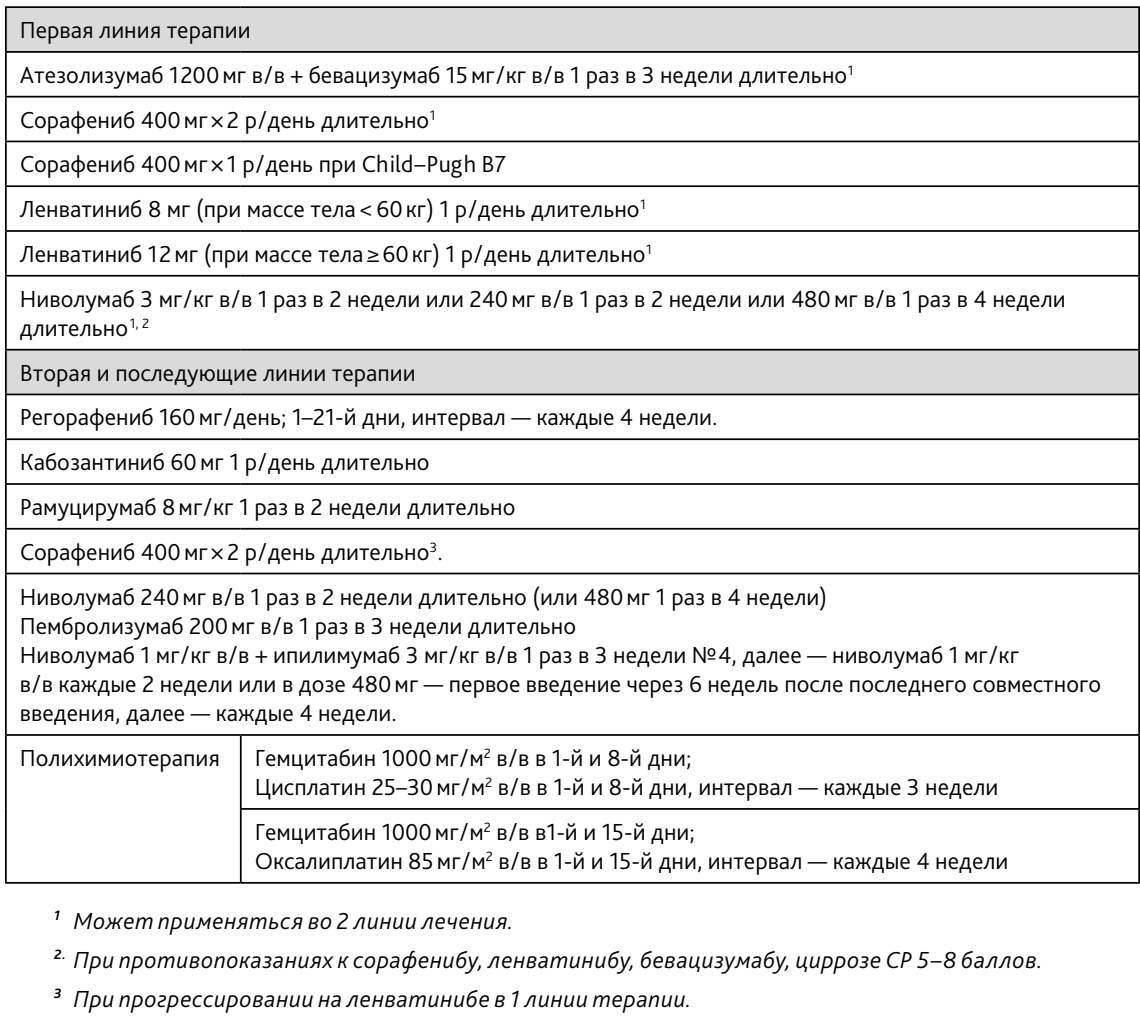

\section{Прогрессирование ГЦР после ортотопической трансплантации печени}

Терапия мультикиназными ингибиторами на фоне необходимой постоянной иммуносупрессивной терапии сопровождается более выраженной токсичностью: сорафениб назначается в начальной дозе 400 мг/день, при удовлетворительной переносимости возможна эскалация до 600-800 мг/день. Переносимость ленватиниба в этой группе больных 
изучена недостаточно; рекомендуется начинать лечение с суточной дозы 8 мг, при хорошей переносимости в течение 10-14 дней доза может быть увеличена до 12 мг/день. Регорафениб и кабозантиниб используются в качестве опций терапии 2-3 линии. Иммунотерапия сопряжена с крайне высоким риском отторжения трансплантата и не используется.

\section{5. Фиброламеллярная карцинома}

Лечение ФЛК, чаще встречающейся у молодых больных без фоновой патологии печени, представляет особую проблему. Основной метод лечения ФЛК-хирургический (включая трансплантацию печени), часто выполняются циторедуктивные операции по удалению первичной опухоли и солитарных внепеченочных метастазов. Эффективность химиоэмболизации неизвестна. Системное лечение метастатической ФЛК проводится с использованием сорафениба, регорафениба, ленватиниба, цитотоксической ХТ на основе платиновых режимов в сочетании с гемцитабином, доксорубицином, капецитабином. ЛТ терапия в ряде случаев позволяет эффективно контролировать локальные проявления/осложнения опухолевого процесса. ФЛК мало чувствительна к терапии ингибиторами контрольных точек иммунного надзора (анти-PD -1/L1).

\section{6. Сопроводительная терапия и лечение фоновой патологии печени}

Пациентам с ГЦР рекомендуется противовирусная терапия сопутствующего хронического гепатита В (ДНК-ВГВ $>2 \times 10^{2} \mathrm{ME} /$ мл) аналогами нуклеот (з) идов (энтекавир или тенофовир) и хронического гепатита С ингибиторами протеазы и полимеразы вируса (глекапревир/пибрентасвир или софосбувир/велпатасвир) одновременно слекарственным или интервенционно-радиологическим лечением независимо от выраженности цитолитического синдрома для улучшения выживаемости пациентов. При активизации хронического вирусного гепатита С (цитолитический синдром: кратное увеличение уровня АСТ/АЛТ) после радикального лечения, после ТАХЭ или на фоне системной терапии возможно одновременное проведение курса лечения прямыми противовирусными агентами у больных с компенсированной функцией печени. Клинически значимых межлекарственных взаимодействий между прямыми противовирусными препаратами и средствами для химиотерапии ГЦР не описано.

Неспецифическая терапия для профилактики лекарственного поражения печени препараты урсодезоксихолевой кислоты (10-15 мг/кг/сут.), S-адеметионина (800 мг/сут.). Назначение препаратов эссенциальных фосфолипидов неэффективно.

\section{7. Особенности ведения и лечения больных ГЦР на фоне цирроза печени}

Сопроводительная корригирующая терапия осложнений фоновой патологии (в том числе цирроза) печени и опухолевого процесса существенно увеличивает выживаемость больных ГЦР. В условиях длительного лекарственного лечения ГЦР может возрастать 
клиническая значимость сопутствующего цирроза печени из-за увеличения выраженности портальной гипертензии, декомпенсации функции органа. Декомпенсация цирроза печени конкурентно влияет на общую выживаемость больных ГЦР, ухудшает переносимость лекарственного лечения вплоть до полной отмены. Необходимо регулярное, не реже 1 раза в месяц, тщательное мониторирование функции печени, проведение активной профилактики и лечение осложнений цирроза печени. При декомпенсированном циррозе - класс Child-Pugh B-C ( $\geq 8$ баллов) - лекарственное лечение ГЦР, как правило, не проводится в связи с ожидаемо высокой токсичностью и отсутствием подтверждения увеличения выживаемости.

Декомпенсация цирроза печени на фоне противоопухолевого лечения иногда ошибочно расценивается как клиническое прогрессирование ГЦР: при отсутствии убедительных признаков прогрессии опухоли необходимо активное лечение осложнений цирроза под наблюдением гастроэнтерологов/гепатологов.

При нарастании проявлений печеночной недостаточности (Child-Pugh $\geq+2$ балла от исходного) противоопухолевое лечение следует приостановить на 10-15 дней, провести активную терапию осложнений цирроза. При положительной динамике лечение может быть возобновлено с редукцией дозы активного препарата.

Стойкое ухудшение функции печени (Child-Pugh B >7 баллов), развитие осложнений цирроза (асцит-перитонит, гепаторенальный синдром, желудочно-кишечное кровотечение) противоопухолевое лечение завершается до клинически значимого улучшения состояния больного с последующим повторной оценкой показаний к терапии ГЦР.

Варикозное расширение вен пищевода/желудка II-III степени предполагает первичную профилактику желудочно-пищеводного кровотечения неселективными $\beta$-адреноблокаторами. Лигирование варикозно расширенных (3 степени) вен проводится при неэффективности медикаментозного лечения, угрозе кровотечения и для вторичной профилактики рецидивного кровотечения. Рекомендуется регулярный - каждые 6 месяцев - эндоскопический контроль состояния вен пищевода/желудка у пациентов с известным диагнозом цирроза печени или тромбозом ствола воротной вены, получающих противоопухолевое лечение ГЦР.

При снижении уровня альбумина плазмы <28г/л на фоне противоопухолевого лечения, до плановой эвакуации значительного (>5 литров) количества асцитической жидкости или после лапароцентеза настоятельно рекомендуется заместительная курсовая терапия $20 \%$ раствором альбумина (100-200 мл/день, № 5-8) до целевого уровня 32-35 г/л. Цирротический асцит хорошо контролируется адекватной диуретической терапией (спиронолактон до 400 мг/д, фуросемид) под контролем электролитов крови.

При циррозе печени нестероидные противовоспалительные препараты используются строго по показаниям в минимально необходимой дозе короткими курсами и с крайней осторожностью.

Больным с декомпенсированной функцией печени (СР В/C >7 баллов) проводится симптоматическая терапия осложнений цирроза и опухолевого процесса. Однако, при выявлении раннего ГЦР (BCLC O/А в «Миланских» критериях) оправдана консультация трансплантолога 